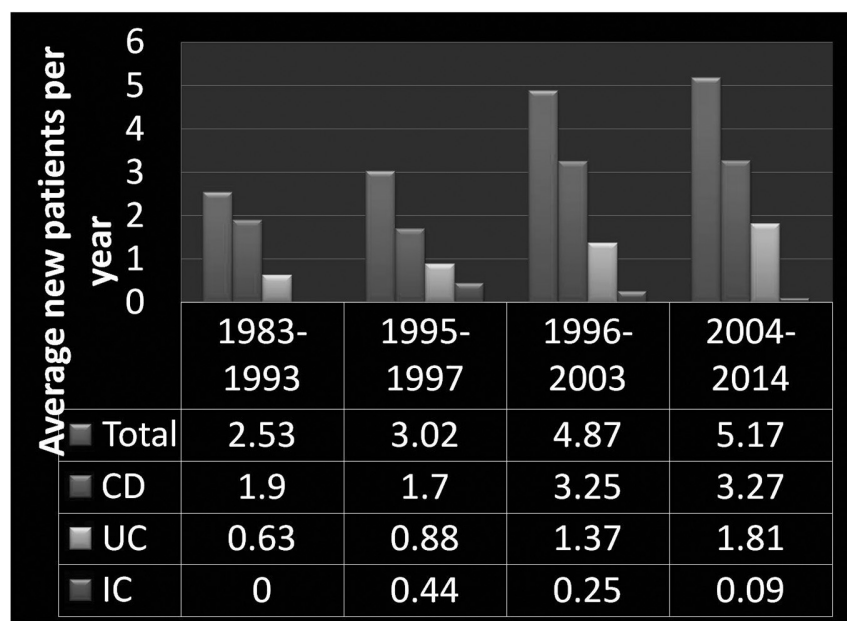

Abstract G353 Figure 1

Introduction The incidence of paediatric inflammatory bowel disease (IBD) has risen significantly across Europe in the last 20 years, although our own data had suggested that this had plateaued by 2004 . We have now studied the data from the same area over the last decade and compared them with our previous studies (1983-2003).

Methods All cases of IBD $<16$ years of age residing in a defined location within our region were prospectively recorded from January 2004 to March 2014. The incidence, age, gender and disease type were analysed and compared to our data from 1983-2003 from the same region.

Results See Table 1. Between 2004 and 2014, there were 57 new patients compared to 39 (1996-2003) and 28 (1983-1993). The overall incidence of IBD was 5.9 per 100,000 per year, Crohn's disease (CD) 3.7 per 100,000 per year and Ulcerative colitis (UC) 2.07 per 100,000 per year compared with 5.4 per 100,000 per year for 1996 to 2003 . There was no statistically significant difference between the two time periods ( $\mathrm{p}$ value of 0.675 ). The median age at diagnosis remains at 12 years with a male to female ratioof 1.7:1.

Conclusion The incidence of paediatric IBD in a defined geographical area within our region has remained similar for more than 15 years with a slight increase in the incidence of UC, suggesting that the previous exponential rise in incidence has reached a stable state.

\section{G354 THE ONGOING RAPID AND SIGNIFICANT RISE OF INCIDENT PAEDIATRIC-ONSET INFLAMMATORY BOWEL DISEASE IN SCOTLAND}

${ }^{1} \mathrm{FA}$ Jagger, ${ }^{2} \mathrm{FL}$ Cameron, ${ }^{3} \mathrm{P}$ Henderson, ${ }^{2} \mathrm{P}$ Rogers, ${ }^{3} \mathrm{P} \mathrm{McGrogan},{ }^{4} \mathrm{~S}$ Loganathan, ${ }^{3}$ RK Russell, ${ }^{3} \mathrm{R}$ Hansen, ${ }^{2} \mathrm{DC}$ Wilson. ${ }^{1}$ University of Aberdeen, Aberdeen, UK; ${ }^{2}$ Child Life and Health, University of Edinburgh, Edinburgh, UK; ${ }^{3}$ Paediatric Gastroenterology, Yorkhill Hospital, Glasgow, UK; ${ }^{4}$ Paediatric Gastroenterology, Royal Aberdeen Children's Hospital, Aberdeen, UK

\subsection{6/archdischild-2015-308599.310}

Background The worldwide incidence of paediatric-onset inflammatory bowel disease (PIBD) is rising, with Scotland having the highest rate in the UK. Scottish PIBD data over the last 40 years has shown a consistent increase, including a $76 \%$ rise over 13 years around the millennium (Henderson P et al. IBD 2012;18:999-1005).
Aims The aim of this study was to calculate current PIBD incidence rates in Scotland and to determine if the temporal trend of significant increase has been maintained.

Methods Historical data from 2003-2008 (cohort 1) was compared to prospective, nationwide data of all incident cases diagnosed in paediatric services (under 16 years of age) from 2009-2013 (cohort 2). Age-sex adjusted incidence rates were calculated using population data from the General Registrar's Office for Scotland. Cases were classified as Crohn's disease (CD), ulcerative colitis (UC) or inflammatory bowel disease unclassified (IBDU) and diagnosed according to the Porto criteria. Statistical analysis was performed using Poisson regression.

Results A total of 436 patients were diagnosed with PIBD over six years in cohort 1 (265 CD, $115 \mathrm{UC}, 56 \mathrm{IBDU})$ compared to 478 children over five years in cohort 2 (286 CD, 126 UC, 66 IBDU). Median age at diagnosis in cohort 2 (60\% males) was 12.3 years, similar to cohort $1(58 \%$ males $)$ at 11.9 years. The adjusted incidence rate increased from 7.8/100,000/year (95\% CI 7.1-8.6) in cohort 1 (2003-2008) to $10.4 / 100,000 /$ year (95\% CI 9.6-11.5) in cohort $2(2009-2013)$ ( $\mathrm{p}<0.001)$. This significant increase was also seen individually for CD (4.7/ 100,000/year [95\% CI 4.2-5.4] compared to 6.3/100,000/year [95\% CI 5.6-7.0] $[\mathrm{p}<0.0001])$ and UC $(2.1 / 100,000 /$ year [95\% CI 1.7-2.5] compared to $2.7 / 100,000 /$ year [95\% CI $2.3-$ 3.3] $[\mathrm{p}=0.009])$. There was a non-significant increase in IBDU from $1.0 / 100,000 /$ year $(95 \%$ CI $0.7,1.3)$ in cohort 1 to $1.4 /$ $100,000 /$ year $(95 \%$ CI $1.1,1.8)$ in cohort 2 ( $p=0.07)$.

Conclusion There continues to be an ongoing rise in incident PIBD (and both CD and UC) in 2009-13 in this national, population-based study compared to recent historical data, with a further significant rise of $33 \%$. The reasons behind this continued increase remain unclear and further research is needed to elucidate potential factors in aetiopathogenesis.

\section{G355 PRESENTING PHENOTYPE OF ULCERATIVE COLITIS (UC) IN CHILDREN 2010-13}

${ }^{1} \mathrm{JJ}$ Ashton, ${ }^{1,2} \mathrm{~T}$ Coelho, ${ }^{2} \mathrm{~S}$ Ennis, ${ }^{1} \mathrm{~A}$ Batra, ${ }^{1} \mathrm{NA}$ Afzal, ${ }^{1} \mathrm{RM}$ Beattie. ${ }^{1}$ Paediatric Gastroenterology, University Hospitals Southampton, Southampton, UK; ${ }^{2}$ Human Genomics, University of Southampton, Southampton, UK

\subsection{6/archdischild-2015-308599.311}

Aims There has been a two-fold increase in the incidence of paediatric-onset UC over the last 20 years; there are few recent reports of the presenting phenotype - symptoms, inflammatory markers and disease extent. We report the presenting features of a defined cohort and compare to previous data.

Methods Patients diagnosed with UC at University Hospitals Southampton from 2010-2013 were identified from an in-house database. Data were obtained from note review using a standardised proforma and compared to previous UK data. ${ }^{1}$ Weight and height at diagnosis are presented as median SDS (95\% CI).

Results 49 children were included. Median age 13.18 (Range 1.59-16.24 years), 27 male. The most common presenting features are seen in Table 1. Weight loss (36.7\%) and lethargy $(36.7 \%)$ were less common. The majority of patients presented with pancolitis $(68.1 \%)$.

A significant number of patients present with normal inflammatory markers (percentage with normal CRP 69.4\%, normal ESR 34.9\%). Median CRP $3.0 \mathrm{mg} / \mathrm{L}(0.0-12-9)$, ESR21.5 mm/ hr (15.2-26.8)). 
Weight SDS was $0.14(-0.20$ to 0.48$)$. Seventeen patients $(39.5 \%)$ were greater than $1 \mathrm{SD}$ above the median. Seven patients $(15.9 \%)$ were between -1 and -2 SD. Height SDS at diagnosis was 0.5315 (0.19 to 0.87 ). Thirty percent were overweight (BMI $>1$ SD above the median) at diagnosis.

Positive family history ( $3^{\text {rd }}$ degree relative or closer) of IBD was seen in $26.5 \%$.

\begin{tabular}{|c|c|c|}
\hline 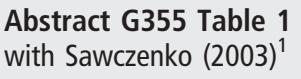 & Comparison of data & a from Ashton (2015) \\
\hline \multicolumn{3}{|c|}{ (Percentage with symptom at presentation) } \\
\hline & Sawczenko & Ashton (Wessex, Southern \\
\hline & (UK) & England) \\
\hline No. patients & 172 & 49 \\
\hline Rectal Bleeding & $84 \%$ & $91.8 \%$ \\
\hline Diarrhoea & $74 \%$ & $91.8 \%$ \\
\hline Abdominal Pain & $62 \%$ & $87.8 \%$ \\
\hline \multicolumn{3}{|c|}{ Triad of Rectal bleeding, Diarrhoea and } \\
\hline Abdominal pain & Unknown & $75.5 \%$ \\
\hline Joint disease & $6.4 \%$ & $8.2 \%$ \\
\hline Height SDS & -0.32 (Mean) & 0.532 (Median) \\
\hline Weight SDS & -0.12 (Mean) & 0.14 (Median) \\
\hline
\end{tabular}

Conclusion Growth delay and raised inflammatory markers are seen in a minority of cases of UC at presentation. The majority of patients present with the classical triad of blood in stools, diarrhoea and abdominal pain however this is not invariable and absence does not preclude a diagnosis of UC.

\section{REFERENCE}

1 Sawczenko A. Presenting features of inflammatory bowel disease in Great Britain and Ireland. Arch Dis Child. 2003;88(11):995-1000

\section{G356 JUVENILE IDIOPATHIC ARTHRITIS AND OTHER AUTOIMMUNE DISEASES IN A NATIONWIDE PAEDIATRIC INFLAMMATORY BOWEL DISEASE COHORT}

${ }^{1}$ VM Merrick, ${ }^{1} \mathrm{P}$ Henderson, ${ }^{2} \mathrm{H}$ Drummond, ${ }^{3} \mathrm{~J}$ Van Limbergen, ${ }^{4} \mathrm{RK}$ Russell, ${ }^{2} \mathrm{~J}$ Satsangi, ${ }^{1} D C$ Wilson. 'Child Life and Health, University of Edinburgh, Edinburgh, UK; ${ }^{2}$ Gastrointestinal Unit, Centre for Genomic and Experimental Medicine, University of Edinburgh, Edinburgh, UK; ${ }^{3}$ Department of Paediatrics, Dalhousie University, Halifax, Canada; ${ }^{4}$ Department of Paediatric Gastroenterology and Nutrition, Royal Hospital for Sick Children (Yorkhill), Glasgow, UK

\subsection{6/archdischild-2015-308599.312}

Aims Autoimmune diseases (AIDs) affect up to $10 \%$ of individuals living in Europe, so are a significant cause of chronic morbidity. High rates of immune-mediated comorbidity and familial clustering suggest that genetic predisposition underlies AI disease susceptibility, yet few clinical studies have defined the prevalence rates of co-morbid AIDs in specific paediatric populations. This study aims to document the occurrence of Juvenile Idiopathic Arthritis (JIA) and other AIDs in a Scotland-wide cohort of paediatric inflammatory bowel disease (PIBD; diagnosed $<17$ years of age) patients.

Methods The Paediatric-onset IBD Cohort and Treatment Study (PICTS) is a nationwide Scottish study of incident and prevalent PIBD patients, collecting a wide range of data, including rigorous phenotyping, with continuous long-term follow-up. The PICTS database was interrogated to identify patients enrolled up to $30 / 06 / 12$ (follow-up to 30/06/14) with a diagnosis of at least one associated AID by last follow-up. Cases believed to be related to use of anti-TNF $\alpha$ treatment were excluded.
Results Of 809 patients in the PICTS cohort, 43 had one or more associated AID, an overall co-morbid immune disease rate of 5.3\%; 49\% (21/43) male. There were 44 AIDs in 43 patients; one patient had dual AIDs (psoriasis [PSOR] and spondyloarthropathy [SPA]) co-existing with IBD. Otherwise, there were 7 cases of JIA, 3 cases of SPA and 9 cases of PSOR. Additionally there were 4 cases of coeliac disease, 2 of thyroiditis and 2 cases of type 1 diabetes. No cases of Systemic Lupus Erythematosus (SLE) were identified. There were 15 cases of autoimmune liver disease (Primary Sclerosing Cholangitis [PSC], Auto-Immune Hepatitis [AIH] and Autoimmune Sclerosing Cholangitis [ASC]) in this cohort, accounting for 35\% of all PIBD-associated AID.

Conclusion Over 5\% of PIBD patients in this large cohort study have associated AIDs. Autoimmune liver disease is the commonest AID in this cohort of PIBD patients, followed by PSOR accounting for $23 \%$; JIA accounted for $16 \%$ of PIBD-associated AID.

\section{G357 CHRONIC RECURRENT MULTIFOCAL OSTEOMYELITIS (CRMO): THE VALUE OF WHOLE BODY MRI DEMONSTRATED BY A SERIES OF 13 ADULT AND 34 PAEDIATRIC PATIENTS}

A Guha, M Brown, B Jacobs. Royal National Orthopaedic Hospital, Stanmore, UK

\subsection{6/archdischild-2015-308599.313}

Aim To assess the role of whole-body MRI (WB MRI) in the diagnosis and management of patients with Chronic Recurrent Multifocal Osteomyelitis (CRMO). CRMO is a benign and noninfective autoinflammatory bone disorder characterised by multiple and recurrent inflammatory bone lesions. No universal diagnostic criteria exist.

Methods Retrospective review of CRMO cases diagnosed at this hospital between 2008 to 2014. Cases were identified from patient records, and clinical information was collated from radiology and histopathology records and individual case notes.

Results Forty seven CRMO patients were identified who had had WB MRI, of these 34 were paediatric patients up to the age of 18 years. The number of WB MRI scans per case ranged from 1 to 5 [mean 1.5]. WB MRI identified multifocal lesions in 30 patients. The clavicle, tibia and femur were most frequently involved. All cases were managed with non-steroidal anti-inflammatory medication or bisphosphonates. No children required steroid or anti-TNF treatment or surgical resection.

Conclusions In the absence of specific diagnostic criteria, WB MRI in combination with clinical assessment can aid in the diagnosis of CRMO. WB MRI has almost entirely replaced bone biopsy in the diagnosis of CRMO at our institution.

\section{G358 A SYSTEMATIC REVIEW TO IDENTIFY THE DEFINITIONS OF RECOVERY FOR PAEDIATRIC PATIENTS WITH CHRONIC FATIGUE SYNDROME (CFS) OR MYALGIC ENCEPHALOMYELITIS (ME) USED IN STUDIES SINCE 1994}

Y Moore, NME Anderson, E Crawley. School of Social and Community Medicine, University of Bristol, Bristol, UK

\subsection{6/archdischild-2015-308599.314}

Aims To describe how recovery from paediatric CFS/ME is defined, how many children recover and how long it takes. 